\title{
Evaluación de posturas forzadas en los puestos de trabajo administrativos del Hospital Básico Guamote (c) (1) (8)(2)
}

\author{
Evaluation of Forced Positions in the Administrative Work Posts of the \\ Guamote Basic Hospital
}

Paola Belén Salazar Montero ${ }^{1}$, Adrián Paul Llerena Asadobay ${ }^{2}$, Evelyn Carolina Villarroel Ponce $^{3}$. Sandy Yomary Riofrío García ${ }^{4}$, \& Nelson Mauricio Moreno Moreira ${ }^{5}$ Recibido: 10-02-2019 / Revisado: 15-02-209 /Aceptado: 04-03-2019/ Publicado: 27-04-2019

\begin{abstract}
.
DOI: https://doi.org/10.32/cienciadigital.v3i1.947

The study is an evaluation of forced postures in the jobs of the administrative area of the "BASIC GUAMOTE HOSPITAL", which have as main activity the coordination, organization and supply of each of the processes under their charge, forcing to stay a large part of their working time in a sitting position, carrying out their daily work activities. In the study, a general description is made of the activities that are carried out in each job, as well as an initial evaluation of the existing risks. To this end, risk factors such as movement frequency, posture duration, trunk postures, neck postures, upper limb postures, lower extremity postures, and identification of forced postures through the application of check list are analyzed. for the identification of forced postures published by the Institute of Safety, Health and Welfare at Work based on the standards UNE-EN 1005-4: 2005 + A1: 2009 and ISO 11226: 2000. by which it was determined that there is a total of 19 forced postures. Subsequently, the risk of suffering
\end{abstract}

\footnotetext{
${ }^{1}$ Master en Prevención de Riesgos Laborales. Médico General. Docente del Instituto Tecnológico Superior Riobamba. Riobamba-Ecuador. paosalazarm88@hotmail.com psalazar@intitutos.gob.ec

2 Médico General. Estudiante de Postgrado en Dirección en Gerencia Hospitalaria -Universidad Internacional de la Rioja. Riobamba-Ecuador. adrianchuspr@hotmail.com @intitutos.gob.ec

${ }^{3}$ Médico General. Docente del Instituto Tecnológico Superior Riobamba. Riobamba - Ecuador. joflim98@hotmail.com evillarroel@intitutos.gob.ec

${ }^{4}$ Médico General. Docente del Instituto Tecnológico Superior Riobamba. Riobamba - Ecuador. yomary_2008@gmail.com sriofrio@intitutos.gob.ec

${ }^{5}$ Médico General. Docente del Instituto Tecnológico Superior Riobamba. Riobamba - Ecuador. morenomauricio@live.com. gmoreno@intitutos.gob.ec
} 
bodily disorders related to work was estimated by adopting forced postures as a result of the activities performed throughout the working day by means of the Rapid Entire Body Assessment method, which has been developed by Hignett and McAtamney. (Nottingham, 2000) obtaining as result that 4 workers present a high average risk, and 3 with high risk, in which the need for intervention is prompt and necessary, Putting measures aimed at preventing the adoption of these forced postures, reducing or eliminating the ergonomic risk found and the position adopted in the workplace due to the adoption of incorrect postures.

Keywords: Forced posture, workers, job position, risk, pathology.

\section{Resumen.}

El estudio se trata de una evaluación de posturas forzadas en los en los puestos de trabajo del área administrativa del "HOSPITAL BASICO GUAMOTE", los cuales tienen como actividad principal la coordinación, organización y abastecimiento de cada uno de los procesos a su cargo, obligándolo a permanecer gran parte de su jornada laboral en posición sentada realizando sus actividades laborales cotidianas. En el estudio se realiza una descripción general de las actividades que se realizan en cada puesto de trabajo, además de una evaluación inicial de los riesgos existentes. Para lo cual se analiza los factores de riesgo como Frecuencia de movimientos, Duración de la postura, Posturas de tronco, Posturas de cuello, Posturas de la extremidad superior, Posturas de la extremidad inferior, e identificar las posturas forzadas mediante la aplicación de check list para la identificación de posturas forzadas publicado por el Instituto de Seguridad, Salud y Bienestar en el Trabajo basado en las normas UNE-EN 1005-4:2005+A1:2009 e ISO 11226:2000. mediante el cual se pudo determinar que existen un total de 19 posturas forzadas. Posteriormente se estimó el riesgo de padecer desórdenes corporales relacionados con el trabajo por adoptar posturas forzadas como resultado de las actividades desempeñadas a lo largo de la jornada laboral mediante el método Rapid Entire Body Assessment) que ha sido desarrollado por Hignett y McAtamney. (Nottingham, 2000) obteniendo como resultado que 4 trabajadores presentan un riesgo medio alto, y 3 con riesgo alto, en el cual la necesidad de intervención es pronta y necesaria, Porponiendo medidas destinadas a prevenir la adopción de estas posturas forzadas, reduciendo o eliminando el riesgo ergonómico encontrado y la postura adoptada en los puestos de trabajo por la adopción de posturas incorrectas.

Palabras claves: Postura forzada, trabajadores, puesto de trabajo, riesgo, patología. 


\section{Introducción.}

En la actualidad las organizaciones gubernamentales se han preocupado por brindar una atención al usuario con calidad y calidez lo que obliga a que éstas solventen servicios en muchas ocasiones sobrepasando las capacidades humanas por las cuales se consumen y desgastan recursos para lograr el liderazgo, sin embargo, el mismo debe ser atribuido al desempeño de los trabajadores, por lo que en la actualidad existe normativa que ampara al trabajador en donde se resaltan estándares que velan la salud y seguridad ocupacional en relación a su puesto de trabajo, según el Real Decreto 488/1997, de 14 de abril, define al puesto de trabajo a lo "constituido por un equipo con pantalla de visualización provisto, en su caso, de un teclado o dispositivo de adquisición de datos, de un programa para la interconexión persona/máquina, de accesorios ofimáticos y de un asiento y mesa o superficie de trabajo, así como el entorno laboral inmediato" (Sociales, 1997). Por todo esto se hace imprescindible la implementación de una cultura basada en la prevención mediante la implantación de un sistema de gestión de seguridad y salud ocupacional, que ha sido un gran paso en nuestro continente, más aun en nuestro país, alcanzando y generando mayor concientización ante la necesidad de implantar estos sistemas e incorporar una iniciativa ergonómica como punto principal ante una prevención de riesgos laborales, permitiendo cambiar la manera de concebir el trabajo humano en las empresas, sin embargo no se logra aun concientizar de esta forma la prevención en la mayor parte de las industrias.

Es así que se debe considerar que al implementar mecanismos de prevención acordes ante los riesgos ergonómicos generados por la adopción de posturas forzadas que se presentan en el puesto de trabajo es un reto importante, ya que existen empresas que disponen de recursos necesarios para equipar a determinados puestos de trabajo de tecnologías modernizadas que obligan a tomar medidas de prevención distintas a una empresa que por otro lado no cuenta con recursos necesarios y que desarrolle sus actividades con maquinaria que ha sobrepasado ya su tiempo de vida útil en muchas ocasiones, lo que obliga a que el trabajador se adapte al puesto, y no viceversa, generado por falta de recursos que impiden que se pueda desarrollar una cultura y una toma de medidas preventivas acorde al puesto de trabajo.

Algunas de estas posturas o movimientos al ser inadecuados o forzados pueden generar problemas para la salud si se realizan con frecuencias altas o durante periodos prolongados de tiempo" (Instituto Nacional de Seguridad, Salud y Bienestar en el Trabajo)) en los trabajadores en relación a sus puestos de trabajo enfocando principalmente a las áreas administrativas del Hospital Básico Guamote.

Es de esta manera que los resultados obtenidos en esta evaluación, servirán de evidencia sobre la problemática y falencias de los puestos evaluados en relación a su diseño, planteando medidas correctivas o de prevención para una correcta implementación de prácticas ergonómicas. Servirá también como una guía para la administración del Hospital ya que una vez evaluada y obtenida la situación actual de los puestos de trabajo con respecto a las 
posturas forzadas adoptadas por él trabajador, se podrá incorporar los cambios que sean necesarios en el plan estratégico institucional, con el compromiso de implementarlo en un corto o mediano plazo y este no sea solo en función de un presupuesto asignado, sino que evidencie una verdadera gestión.

La evaluación de posturas forzadas se realizará mediante la aplicación de check list para la identificación de posturas forzadas publicado por el Instituto de Seguridad, Salud y Bienestar en el Trabajo basado en las normas UNE-EN 1005-4:2005+A1:2009 e ISO 11226:2000 así como también el método Rapid Entire Body Assessment que ha sido desarrollado por Hignett y McAtamney. (Nottingham, 2000).

\section{Marco Teorico}

\section{Descripción de puestos}

- Director

Cumple funciones de dirigir el buen funcionamiento global del hospital como máxima autoridad y representante legal en base a la normativa legal vigente, por lo que la actividad que realiza es el despacho de documentación en digital por el lapso de 6 horas diarias en posición sentada

\section{- Bioquímico farmacéutico}

La función que desempeña es realizar el despacho de documentación, registro de entrega de recetas prescritas por el personal médico, informes de gestión y consumo, actas, inventario, solicitud de insumos y medicamentos, de tal manera permanece en su puesto de trabajo en posición sentada durante las 7 horas diarias y 1 hora diaria en supervisión al personal operativo bajo su cargo, laborando en total 8 horas diarias.

\section{- Asistente de admisiones}

La función que desempeña es realizar la apertura de historias clínicas, despachar asuntos pendientes estadísticos así como de la recolección de los mismos del personal médico, producción hospitalaria, de tal manera permanece en posición sentada en su puesto de trabajo 6 horas diarias y en posición bípeda las 2 restantes, laborando un total de 8 horas diarias

- Atención al usuario

Asesoría sobre los procesos de tramites ciudadanos del Ministerio de Salud realizando estas actividades por un tiempo de 6 horas en posición sentada y 2 horas de pie laborando un total de 8 horas diarias.

- Guardalmacén 
La función que desempeña es realizar actividades de recepción, supervisión, entrega y despacho de medicamentos, dispositivos médicos, materiales de oficina y limpieza durante 8 horas diarias, el profesional ejerce actividades de carga de paquetería 3 horas diarias durante el día de mayor producción, desempeña también un aproximado de 5 horas de actividades administrativas en posición sentada.

- Activos Fijos

Registra los movimientos de los bienes, constataciones en las diferentes áreas del hospital e informes sobre actividades y registro en digital, por lo que desarrolla sus actividades durante las 4 horas diarias en bipedestación y otras 4 horas en sedestación.

\section{- Analista de Talento Humano}

Realiza funciones administrativas como administrar, seleccionar y desarrollar el talento humano del hospital, despachar documentación, realizar informes técnicos sobre las necesidades de la institución correspondiente al talento humano casi un total de 8 horas en posición sentada.

Las posturas forzadas en varias ocasiones dan origen a trastornos musculo esqueléticos, síntomas que son de aparición lenta y de apariencia insidiosa por lo que se suele ignorar el síntoma hasta que se transforma en un trastorno crónico generando un daño permanente, manifestadas en zonas anatómicas como: tejido conectivo, tendones y sus vainas, nervios, además de impedir o disminuir el flujo sanguíneo a través del paquete arterio - venoso.

Las afecciones más frecuentes aparecen en la zona de hombros y cuello manifestada en molestias, incomodidad, impedimento o dolor persistente en articulaciones, músculos, tendones y otros tejidos blandos, con o sin manifestación física, causado o agravado por movimientos repetidos, posturas forzadas y movimientos que desarrollan grandes esfuerzos. (Sagrario Cilveti Gubía, Víctor Idoate García., 2000)

Cilveti y García (2000) manifiestan que "aunque las lesiones dorso lumbares y de extremidades se deben principalmente a la manipulación de cargas, también son comunes en otros entornos de trabajo, en los que no se dan manipulaciones de cargas y sí posturas inadecuadas con una elevada carga muscular estática."(p.13) Para lo cual definen tres etapas de aparición de los trastornos originados por posturas forzadas, en la que en la primera etapa aparece dolor y cansancio durante las horas de trabajo, desapareciendo fuera de éste. Esta etapa puede durar meses o años. A menudo se puede eliminar la causa mediante medidas ergonómicas, en la segunda etapa, los síntomas aparecen al empezar el trabajo y no desaparecen por la noche, alterando el sueño y disminuyendo la capacidad de trabajo. Esta etapa persiste durante meses y durante la tercera etapa, los síntomas persisten durante el descanso. Se hace difícil realizar tareas, incluso las más triviales. 
Por tanto el INSSBT establece que los trastornos musculo esqueléticos (TME) más frecuentes en la extremidad superior son: tendinitis del manguito de los rotadores, epicondilitis, epitrocleitis, síndrome del túnel carpiano y ganglión.

\section{Traumatismos específicos en mano y muñeca}

Tendinitis: es una inflamación de un tendón debida, entre otras causas, a que está repetidamente en tensión, doblado, en contacto con una superficie dura o sometida a vibraciones. Como consecuencia de estas acciones el tendón se ensancha y se hace irregular. (Sagrario Cilveti Gubía, Víctor Idoate García., 2000).

Tenosinovitis: producción excesiva de líquido sinovial por parte de la vaina tendinosa, que se acumula, hinchándose la vaina y produciendo dolor. Se originan por flexiones y/o extensiones extremas de la muñeca. (Sagrario Cilveti Gubía, Víctor Idoate García., 2000)

Dedo en gatillo: se origina por flexión repetida del dedo, o por mantener doblada la falange distal del dedo mientras permanecen rectas las falanges proximales. (Sagrario Cilveti Gubía, Víctor Idoate García., 2000)

Síndrome del canal de Guyon: se produce al comprimirse el nervio cubital cuando pasa a través del túnel Guyon en la muñeca. Puede originarse por flexión y extensión prolongada de la muñeca, y por presión repetida en la base de la palma de la mano. (Sagrario Cilveti Gubía, Víctor Idoate García., 2000

Síndrome del Túnel Carpiano Definición: Corresponde a la compresión del nervio mediano a su paso por la muñeca a nivel del interior del túnel del carpo. (INSSBT, Síndrome del Túnel Carpiano)

Ganglión: Un ganglión o quiste sinovial corresponde a una protrusión (salida) del líquido sinovial a través de zonas de menor resistencia de la cápsula articular de la muñeca (huesos del carpo) o de las vainas sinoviales de los tendones. (INSSBT, Ganglion )

\section{Trastornos de hombro y brazo}

Tendinitis del manguito de los rotadores: Los trastornos aparecen en trabajos donde los codos deben estar en posición elevada, o en actividades donde se tensan los tendones o la bolsa subacromial. Se asocia con acciones repetidas de levantar y alcanzar con y sin carga, y con un uso continuado del brazo en abducción o flexión. (Sagrario Cilveti Gubía, Víctor Idoate García., 2000). 
La Epicondilitis o "codo de tenista": es una lesión por esfuerzo repetitivo en el movimiento de pronación-supinación forzada, en la que se inflaman los tendones de los músculos de la cara externa del codo (los músculos extensores de los dedos y la muñeca, y los supinadores del antebrazo) con un origen común (unión) en el Epicóndilo (INSSBT, Epicondilitis).

Epitrocleitis: La Epitrocleitis o "codo del golfista" es una lesión por esfuerzo repetitivo en el movimiento de supinación forzada. Los tendones de los músculos del primer plano del antebrazo ventral, que presentan un origen en la Epitróclea (Epicóndilo medial), se inflaman por incremento de la tensión. (INSSBT, Epitrocleitis)

\section{Trastornos de la columna}

Síndrome cervical por tensión: Corresponde a un cuadro clínico doloroso producido por una contractura muscular incontrolable y persistente en la región cervical posterior, que afecta a un músculo o a un grupo muscular. (INSSBT, Síndrome cervical por tensión ).

Lumbalgia aguda o crónica: La lumbalgia es una contractura dolorosa y persistente de los músculos que se encuentran en la parte baja de la espalda, específicamente en la zona lumbar, siendo muy común en la población adulta. (INSSBT, Lumbalgia )

\section{Traumatismos de miembros inferiores}

Bursitis prepatelar: Corresponde a una inflamación de la cara anterior de la rodilla. (INSSBT, Bursitis prepatelar).

\section{Metodologia.}

El presente estudio es una investigación aplicada ya que el problema surge directamente de la práctica social y genera resultados que pueden aplicarse (son aplicables y tienen aplicación en el ámbito donde se realizan) (PANEQUE, 1988)

Es una investigación explicativa observacional de esta manera se organiza la observación de datos de manera tal que nos permita también verificar o refutar hipótesis, partiendo de problemas bien identificados en los cuales es necesario el conocimiento de relaciones causaefecto. (PANEQUE, 1988)

Siendo además un estudio transversal realizando el estudio de enfermedades o factores de riesgo. Existen varios métodos que permiten la evaluación del riesgo asociado a la carga postural, los cuales se diferencian por el ámbito de aplicación, así como la evaluación de posturas individuales o por conjuntos de posturas, los condicionantes para su aplicación o por las partes del cuerpo evaluadas o consideradas para su evaluación. El método REBA es uno de los métodos observacionales para la evaluación de posturas más desarrollado en la 
práctica. De manera general el método REBA está basado en el método RULA, diferenciándose principalmente en la integración durante la evaluación de las extremidades inferiores.

Se aplica también el Check list del Instituto Nacional de Seguridad, Salud y Bienestar en el Trabajo (INSSBT) para la identificación de posturas forzadas, el mismo que está basado en las normas UNE-EN 1005-4:2005+A1:2009 e ISO 11226:2000. El mismo que nos permitirá identificar de manera más rápida y sencilla los factores de riesgo a ser valorados también por el método REBA.

Los puestos administrativos involucrados en este estudio son los puestos de trabajo que laboran de 6 a 8 horas diarias a partir de las 08:00 a las 17:00, incluida la hora de almuerzo, además son puestos incluidos en la Estructura Orgánica de Hospitales Regulados por el Ministerio de Salud Pública donde se identifican como puestos administrativos. Los 7 puestos administrativos a tomarse en cuenta en este estudio son:

Tabla 1. Puestos de trabajo Administrativos del Hospital Básico Guamote

\begin{tabular}{|c|c|c|}
\hline DESCRIPCIÓN DEL PUESTO & $\begin{array}{ll}\mathbf{N}^{\circ} & \mathbf{D E}\end{array}$ & ADMINISTRATIVO \\
\hline & TRABAJADORES & \\
\hline Director/a de Hospital Básico & 1 & $\mathrm{X}$ \\
\hline Químico / Bioquímico Farmacéutico & 1 & $\mathrm{X}$ \\
\hline Asistente de admisiones & 1 & $\mathrm{X}$ \\
\hline Asistente de Atención al Usuario & 1 & $\mathrm{X}$ \\
\hline Analista de Talento Humano & 1 & $\mathrm{X}$ \\
\hline Guardalmacén & 1 & $\mathrm{X}$ \\
\hline Analista de Activos Fijos 1 & 1 & $\mathrm{X}$ \\
\hline TOTAL & 7 & 7 \\
\hline
\end{tabular}

Fuente: Unidad de Talento Humano Hospital Básico Guamote, 2017

La muestra establecida para el estudio es del total de la misma, siendo esta de 7 trabajadores de los puestos administrativos del hospital. 


\section{CHECK LIST PARA LA IDENTIFICACIÓN DE LAS POSTURAS DE TRABAJO} FORZADAS

Para la aplicación de este check list debemos tomar en cuenta los factores de riesgo que son los siguientes:

$\checkmark \quad$ Frecuencia de movimientos: A mayor frecuencia, el riesgo puede aumentar debido a la exigencia física que requiere el movimiento a cierta velocidad.

$\checkmark$ Duración de la postura: El mantener la misma postura durante un tiempo prolongado es un factor de riesgo a minimizar.

$\checkmark$ Posturas de tronco: adoptar las posturas de flexión de tronco, la rotación axial y la inclinación lateral por encima de los límites aceptables de articulación, puede comportar un nivel importante de riesgo.

$\checkmark$ Posturas de cuello: Las posturas de cuello que se deben identificar son la flexión de cuello (hacia adelante), extensión de cuello, inclinación lateral y rotación axial ya que están vinculadas a la observación de los elementos que están fuera del campo de observación directo.

\section{$\checkmark$ Posturas de la extremidad superior :}

$\checkmark \quad$ Brazo (Hombro): Las posturas que influyen en aumentar el nivel de riesgo, si están en el límite de su rango articular son la abducción, la flexión, extensión, rotación externa, y la aducción.

$\checkmark$ Codo: Las posturas o movimientos del codo que pueden llegar a ser forzados son la flexión, la extensión, la pronación y la supinación.

$\checkmark \quad$ Muñeca: La flexión, la extensión, la desviación radial o cubital son posturas que si se realizan de forma forzada durante un tiempo considerable, pueden repercutir en un nivel de riesgo significativo.

$\checkmark \quad$ Posturas de la extremidad inferior: La extremidad inferior incluyendo la cadera y las piernas, tiene movimientos como la flexión de rodilla, flexión de tobillo, dorsiflexión del tobillo.

\section{Metodología de evaluación Reba}

Esta metodología agrupa el cuerpo en segmentos, es decir, Grupo A: piernas, tronco y cuello, Grupo B: brazos, antebrazos y muñecas, obteniendo cada uno su puntuación, posteriormente se obtiene una puntuación final, la misma que se modifica según el tipo de actividad muscular desarrollada sean estos movimientos repetitivos, posturas estáticas o cambios de postura importantes. El resultado determina el nivel de riesgo de padecer lesiones estableciendo el nivel de acción requerido y la urgencia de la intervención, cuanto mayor sea el valor del resultado mayor será el riesgo previsto de la postura adoptada, es decir, si el valor indica 1, 
el riesgo será inapreciable, mientras que el valor indica 15, se trata de una postura de riesgo muy alto sobre la que se debe actuar de inmediato. (Trabajo, Análisis de posturas forzadas

Figura 1. Evaluación REBA Grupo A NTP 601, 2001

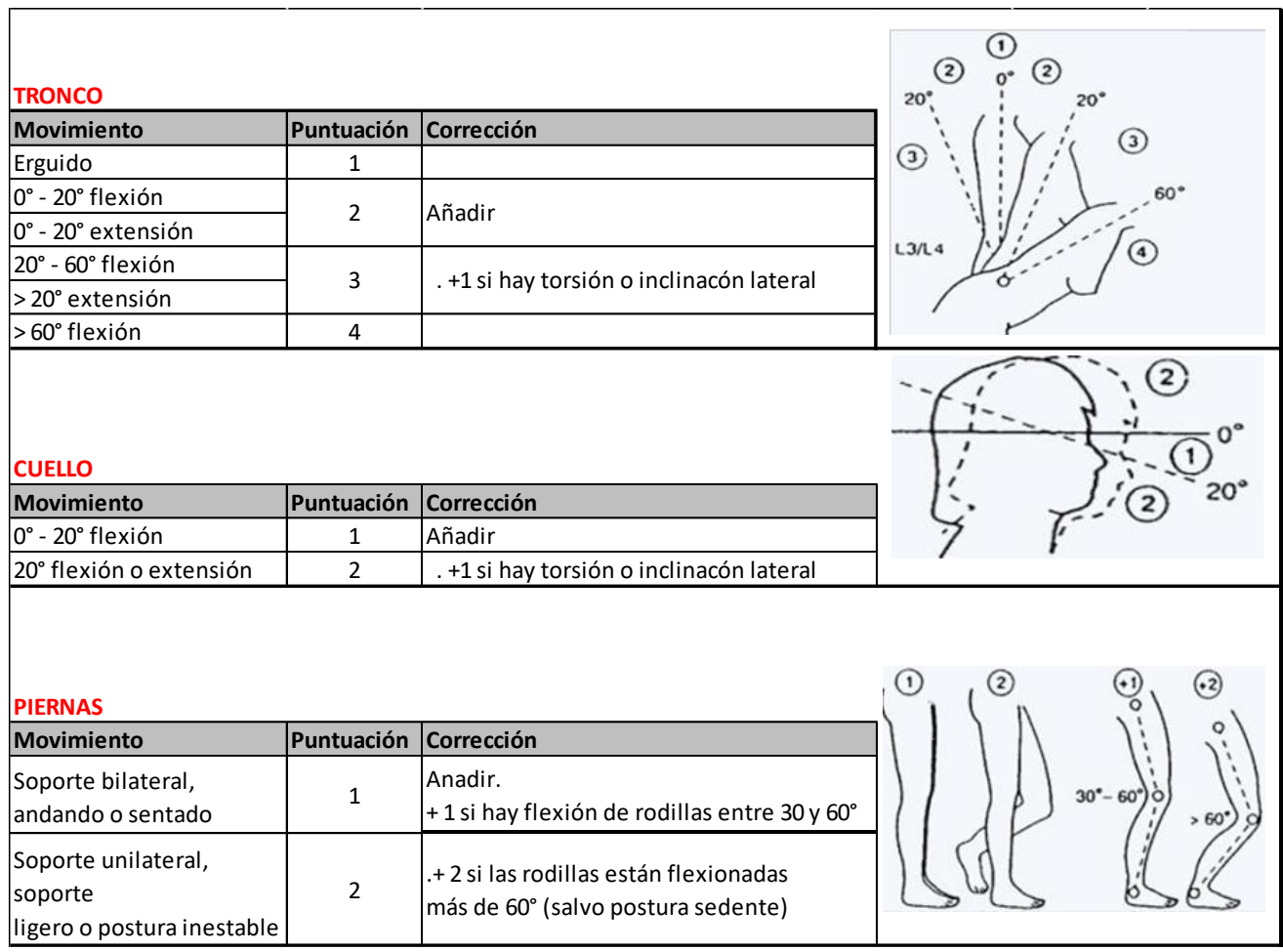

Figura 2. Evaluación REBA Grupo B NTP 601, 2001

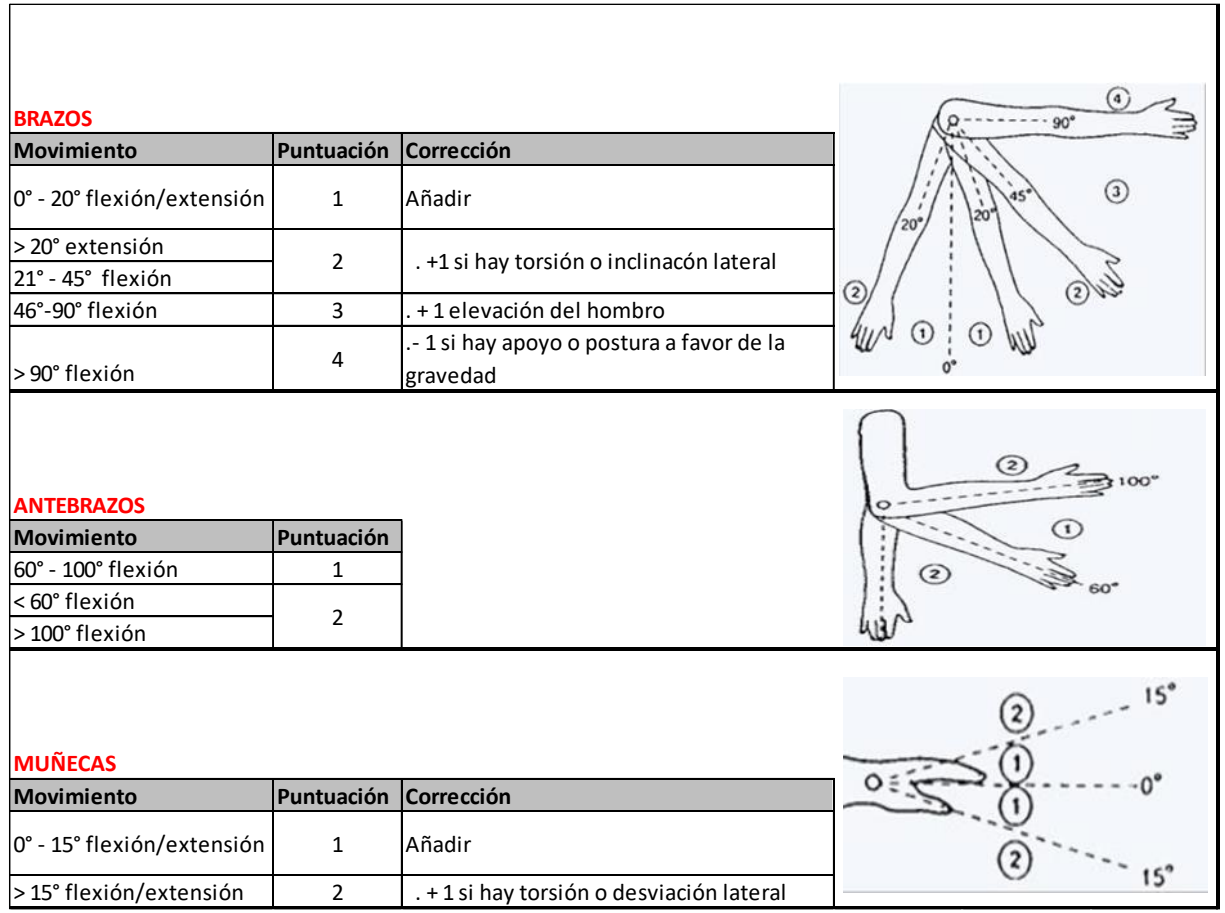


El grupo A tiene un total de 60 combinaciones posturales para el tronco, cuello y piernas. La puntuación obtenida de la tabla A estará comprendida entre 1 y 9; a este valor se le debe añadir la puntuación resultante de la carga/ fuerza cuyo rango está entre 0 y 3 . (Tabla 11) (Cuixart, 2001) VER ANEXO 2.

El grupo B tiene un total de 36 combinaciones posturales para la parte superior del brazo, parte inferior del brazo y muñecas, la puntuación final de este grupo, tal como se recoge en la tabla B, está entre 0 y 9; a este resultado se le debe añadir el obtenido de la tabla de agarre, es decir, de 0 a 3 puntos. (Tabla 12) (Cuixart, 2001) VER ANEXO 2.

Los resultados A y B se combinan en la Tabla C para dar un total de 144 posibles combinaciones, y finalmente se añade el resultado de la actividad para dar el resultado final REBA que indicará el nivel de riesgo y el nivel de acción. (Tabla13) (Cuixart, 2001) VER ANEXO 2.

\section{La puntuación que hace referencia a la actividad (+1) se añade cuando:}

- Una o más partes del cuerpo permanecen estáticas: por ejemplo, sostenidas durante más de 1 minuto.

- Repeticiones cortas de una tarea: por ejemplo, más de cuatro veces por minuto (no se incluye el caminar).

- Acciones que causen grandes y rápidos cambios posturales.

- Cuando la postura sea inestable.

\section{Puntuación final:}

A las 144 combinaciones posturales finales hay que sumarle las puntuaciones correspondientes al concepto de puntuaciones de carga, al acoplamiento y a las actividades; ello nos dará la puntuación final REBA que estará comprendida en un rango de 1-15, lo que nos indicará el riesgo que supone desarrollar el tipo de tarea analizado y nos indicará los niveles de acción necesarios en cada caso. (Tabla 14) (Cuixart, 2001) VER ANEXO 2

Para realizar un registro de la puntuación obtenida mientras se evalúa con el método REBA, se utilizará la Hoja de puntuación. Adaptado de Hignett, S., McAtamney, L. (2000) Applied Ergonomics, 31, 201-5, que se demuestra a continuación: (Tabla 15) (Cuixart, 2001) VER ANEXO 2

Todo este procedimiento se realizará para cada puesto de trabajo, y una vez obtenida las 7 evaluaciones, se procederá a realizar la tabulación de los datos obtenido

\section{Resultados.}

El presente estudio trata de realizar una evaluación de las posturas forzadas que adopta el trabajador en su puesto de trabajo el mismo que servirá para plantear soluciones a los 
problemas más comunes en el entorno laboral del trabajador, relacionado con los riesgos ergonómicos. La actividad administrativa en los diferentes puestos administrativos del Hospital Básico Guamote exige un trabajo comprometido y extenuante en muchas ocasiones que sobrepasa la jornada laboral normal. El desarrollo de actividades para brindar una atención médica oportuna de calidad al usuario del Hospital, exige al trabajador mayor esfuerzo físico y carga mental, lo que nos obliga a precautelar el estado de salud del trabajador, sumado a esto, la queja de los trabajadores sobre las molestias causadas por las actividades que realizan diariamente, en condiciones que al parecer no son adecuadas. De esta manera surge el compromiso y la necesidad del cuidado del trabajador a través de la realización de un estudio ergonómico, enfocado especialmente el análisis de posturas forzadas realizadas en los puestos de trabajo del área administrativa del Hospital Básico Guamote.

Debido a una cultura ergonómica casi ausente en nuestro país, sea por desconocimiento o poca voluntad de las empresas en cumplir con las exigencias de la normativa legal existente, pocos recursos asignados, instituciones que no cuentan con recursos preventivos, sistemas de seguridad y salud ocupacional medianamente desarrollados, situación que obliga a que el empresario y el trabajador de importancia a investigaciones como la del presente estudio, mediante el cual se permita dar a conocer a los trabajadores los factores que afectan e influyen en sus actividades laborales o que agravan las patologías que padecen, generando de esta manera el planteamiento de soluciones preventivas cuyo objetivo sea eliminar y reducir los riesgos ergonómicos por posturas forzadas o repetitivas, las mismas que dan como resultado un ausentismo laboral, enfermedades profesionales, y hasta problemas legales que se asocian a la falta de implementación de un sistema de gestión en seguridad y salud.

En nuestro caso, no se cuenta con estadísticas claras sobre bajas laborales o accidentes de trabajo ya que no está implantado un sistema de Gestión de seguridad y salud, por tanto los datos con los que se cuenta son de trabajadores que sufren de patologías agudas o crónicas como hernia discal, lumbalgia, epicondilitis, artrosis de rodilla, migraña, las cuales agravan la situación ergonómica. 
Figura 3. Principales causas de absentismo en el Hospital Básico Guamote

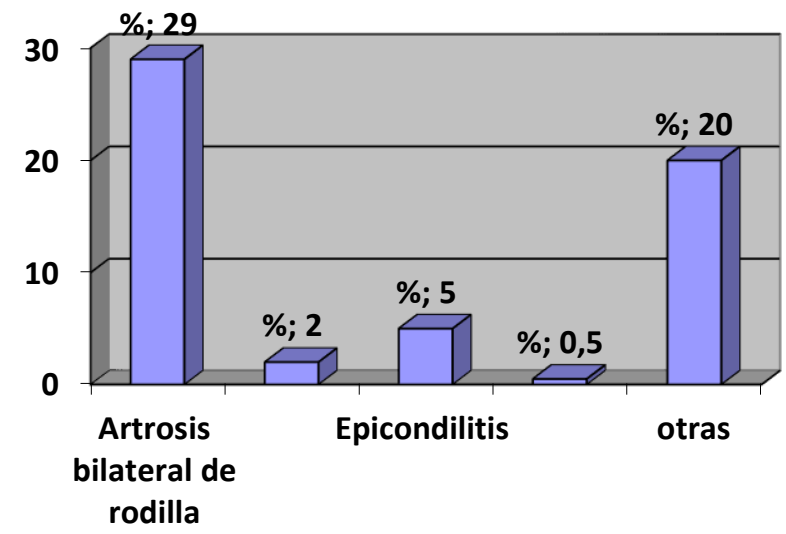

Elaboración; Autores

La poca información documentada existente sobre las bajas laborales nos dan a conocer que la artrosis bilateral de rodilla presenta un absentismo del $29 \%$, patologías como migraña presentan un absentismo del 2\%, los casos de epicondilitis presentan un $5 \%$ de absentismo laboral, accidentes de trabajo sin bajas $2 \%$, lumbalgia $0.5 \%$, dos accidentes in itinere causaron un absentismo del $20 \%$ y otras causas de absentismo el $20 \%$.

No existen datos sobre investigación ni seguimiento relacionado a este ausentismo, es a razón de lo cual el presente estudio nos permitirá iniciar la implantación de un adecuado Sistema de Seguridad y Salud Ocupacional, ya que hasta finales de 2017 solo se contaba con un médico ocupacional, posterior a esta fecha se cuenta ya con un Técnico en Prevención de Riesgos laborales que colabora con el Hospital a tiempo parcial.

Una vez realizada la evaluación mediante la observación del trabajo realizado en el puesto durante un tiempo representativo, de ha tabulado los siguientes resultados:

Se puede determinar que mediante la aplicación del check list existen 19 posturas forzadas encontradas en los trabajadores, las posturas más incidentes son: inclinación de la cabeza y del tronco hacia adelante, en donde los puestos de Asistente de admisiones y Bioquímico farmacéutico son los que cuentan con mayor cantidad de posturas forzadas observadas. Además, se evidencia que un trabajador (asistente de atención al usuario) no cuenta con una silla ergonómica.

Siendo este un método demasiado subjetivo y por tanto complejo, sujeto a criterio del evaluador. Por tanto, se quiere puntualizar que las posturas forzadas identificadas fueron: 
Tabla 1. Resumen de Posturas Identificadas mediante Check List

\section{Postura forzada}

\section{Total Trabajador que Presenta La Postura Forzada}

\section{Cabeza en postura forzada:}

Girada

Inclinada hacia delante (en flexión acusada)

Inclinada hacia los lados

Tronco en postura forzada:

Girado

Inclinado hacia delante (en flexión acusada)

Brazo (hombro) en postura forzada:

Brazo levantado hacia delante (flexión acusada)

\section{Mano (muñeca) en postura forzada:}

Desviación radial de la mano

\section{Extremidad inferior en postura forzada}

\section{Postura forzada}

Rodillas muy extendidas estando sentado ( $\sin$ inclinar el tronco hacia atrás)

Total
2 Asistente De Admisiones

4 Director, Bioquímico. Guardalmacén, Analista De Talento Humano

1 Bioquímico

1 Asistente De Admisiones

4 Director, Bioquímico. Guardalmacén, Analista De Talento Humano

4 Director, Bioquímico, Asistente De Admisiones, Analista De Talento Humano

2 Asistente De Atención Al Usuario, Analista De Talento Humano

\section{Total Trabajador que Presenta La Postura} Forzada

$1 \quad$ Asistente De Admisiones

19

Elaboracion; Autores 
Este método nos permitió evaluar posturas que no se toman en cuenta en el método REBA por tanto nos permitió valorar específicamente más factores de riesgo en lo que corresponde a miembros inferiores como es el tobillo, sin tomar en cuenta agarre, fuerza o si se cuenta con apoyo o gravedad. Por lo que definitivamente es más subjetiva que el método REBA.

\section{RESULTADOS OBTENIDOS MEDIANTE LA APLICACIÓN DEL MÉTODO REBA}

Una vez realizada la evaluación por el método REBA se puede determinar que es un método de fácil aplicación ya que tiene una estructura más técnica que el check list aplicado anteriormente, se siguió el procedimiento indicado en párrafos anteriores, integrando además en la puntuación actividades en donde las partes del cuerpo permanecen estáticas durante más de 1 minuto, excluyendo puntuación por fuerza y agarre. Obteniendo los siguientes resultados:

Tabla 2. Resultados Método REBA

\begin{tabular}{|c|c|c|c|c|}
\hline $\begin{array}{l}\text { DESCRIPCIÓN } \\
\text { DEL PUESTO }\end{array}$ & $\begin{array}{c}\mathbf{N}^{\circ} \text { DE } \\
\text { TRABAJADORES }\end{array}$ & $\begin{array}{l}\text { PUNTUACION } \\
\text { FINAL }\end{array}$ & $\begin{array}{l}\text { NIVEL } \\
\text { DE } \\
\text { RIESGO }\end{array}$ & INTERVENCION \\
\hline $\begin{array}{l}\text { Director/A De } \\
\text { Hospital Básico }\end{array}$ & 1 & 6 & Medio & Necesario \\
\hline $\begin{array}{l}\text { Químico / } \\
\text { Bioquímico } \\
\text { Farmacéutico } 1\end{array}$ & 1 & 9 & Alto & Necesario \\
\hline $\begin{array}{l}\text { Asistente De } \\
\text { Admisiones }\end{array}$ & 1 & 8 & Alto & Necesario \\
\hline $\begin{array}{l}\text { Asistente De } \\
\text { Atención Al } \\
\text { Usuario }\end{array}$ & 1 & 5 & Medio & Necesario \\
\hline Guardalmacén & 1 & 5 & Medio & Necesario \\
\hline $\begin{array}{l}\text { Analista De } \\
\text { Activos Fijos } 1\end{array}$ & 1 & 5 & Medio & Necesario \\
\hline $\begin{array}{l}\text { Analista De } \\
\text { Talento Humano } 1\end{array}$ & 1 & 8 & Alto & Necesario \\
\hline
\end{tabular}

Elaboración; Autores 
Se evidencia en la tabla que las puntuaciones obtenidas en su mayoría van de 5 a 6 equivalentes a un nivel de riesgo medio con una intervención necesaria, los puestos que generan menor riesgo son los puestos de Atención al Usuario, Guardalmacén y Activos Fijos con un puntaje de 5 donde la intervención es necesaria, 1 puesto de trabajo que posee el puntaje de 6 cuya intervención de igual manera es necesaria, sin embargo se evidencia la existencia de tres trabajadores que han obtenido un riesgo alto, que son: el Bioquímico farmacéutico, el Analista de Talento Humano con un puntaje de 8 y el Asistente de Admisiones, obteniendo una puntuación de 9 cuya intervención es pronta y necesaria.

Tabla 3. Resumen de Resultados

\begin{tabular}{|c|c|c|c|}
\hline $\begin{array}{l}\text { NIVEL DE } \\
\text { RIESGO }\end{array}$ & $\begin{array}{l}\mathrm{N}^{\circ} \text { DE } \\
\text { PUESTOS } \\
\text { DE } \\
\text { TRABAJO }\end{array}$ & $\begin{array}{l}\% \text { DE } \\
\text { PUESTOS DE } \\
\text { TRABAJO }\end{array}$ & $\begin{array}{l}\mathrm{N}^{\circ} \mathrm{DE} \\
\text { TRABAJADORES }\end{array}$ \\
\hline INAPRECIABLE & 0 & 0 & 0 \\
\hline BAJO & 0 & 0 & 0 \\
\hline MEDIO ALTO & 4 & 70 & 4 \\
\hline MUY ALTO & 3 & 30 & 3 \\
\hline TOTAL & 7 & 100 & 7 \\
\hline
\end{tabular}

Elaboración; Autores

En este apartado se intenta recopilar los resultados obtenidos durante la evaluación con el método REBA asociando a cuantos trabajadores se verían afectados según el nivel de riesgo, existiendo 5 puestos de trabajo con un riesgo medio alto, causado por posturas forzadas con un puntaje mayor en el grupo A que en el B, es decir, la puntuación más alta se obtuvo en tronco, cuello y piernas. Dos puestos de trabajo presentan un riesgo muy alto, lo que genera riesgo para dos trabajadores, sin evidenciarse riesgos inapreciables o bajos. 


\section{Conclusiones.}

- En el presente trabajo se pudo concluir que no existe un empoderamiento de las autoridades para una adecuada implementación y desarrollo de un Sistema de Gestión de Seguridad y salud ocupacional, lo que ha generado la ausencia de datos estadísticos claros para ser optimizados en el presente estudio, además de diseños de puestos de trabajo inadecuados, que no nos permitió realizar unas tomas fotográficas adecuadassin embargo, se pudo realizar la evaluación mediante la observación.

- Existe deficiencia en lo que corresponde a capacitaciones y formación de cada trabajador, ya que no existe un plan de capacitaciones ni documentos que muestren que los trabajadores del área administrativa del Hospital Básico Guamote cuenten con una formación básica como lo exige la normativa.

- Las posibilidades de implementación de un adecuado sistema de gestión, así como del plan de prevención realizado en el presente estudio son altas, ya que el coste económico es bajo, es decir, de 1,193.76€. Por lo tanto, la implementación en primer lugar se daría con la formación y capacitación de los trabajadores, simultáneamente se prevendría la adopción de posturas forzadas por malos hábitos posturales que se observaron en los trabajadores, resolviendo la problemática mayoritaria, y como tercera fase se daría la implementación del equipamiento ergonómico necesario. Obviamente teniendo en cuenta los tiempos establecidos para cada trabajador según la necesidad de intervención.

- Se pudo obtener resultados en los cuales se evidencia que un $70 \%$ de los puestos evaluados necesitan una intervención con un riesgo medio alto y un $30 \%$ con un riesgo alto haciendo que la necesidad de intervención sea pronta, por tanto, las medidas propuestas son de fácil aplicación y que obtendrán grandes resultados, siendo la misma prioridad.

- En general las medidas preventivas a tomarse son capacitación, formación para que el trabajador a pesar de tener un puesto con un diseño adecuado, no adopte una postura incorrecta, además se debe implementar pausas activas por cada hora de trabajo la pausa se daría durante 15 minutos, ejercicios de elongación y calentamiento, cambio de mobiliario ergonómico en dos puestos de trabajo como son en el puesto de asistente de admisiones es necesario un escritorio en forma de L y en el puesto de atención al usuario es necesario una silla ergonómica. En los demás puestos observados las medidas de prevención giran en torno a un teclado corrediza, mousse inalámbrico, lo que evitaría la posición inclinada del tronco y cabeza.

- La viabilidad de las medidas propuestas es buenas y factibles ya que la prevención de muchas de las posturas adoptadas por el trabajador son posturas que no dependen del diseño del puesto de trabajo, si no de la elección del trabajador. Por tanto, con una capacitación donde se informe las medidas de prevenirlas y de evitarlas, se resolvería dicha problemática.

- Se debe realizar gestión para obtener los recursos económicos necesarios para adquirir el mobiliario y equipamiento faltante, considerando esto como una limitación.

- Una vez capacitado el personal administrativo, se debería continuar realizado el estudio con el personal operativo ya que ejercen actividades que deben ser sometidas a esta evaluación, de esta manera todos los puestos de trabajo estarían sometidos a 
evaluación y se podría conocer cuál es el impacto ergonómico total y el nivel de riesgo en toda la institución. Logrando objetivos a mayor escala.

- Se puede concluir además que estudios como los realizados en este documento, aportan de manera significativa en el desarrollo de la institución objeto de estudio, ya que se demuestra que no es necesario una inversión extremadamente alta, pero si se necesita del verdadero compromiso de autoridades y trabajadores que se preocupen y le brinden la importancia necesaria a cuidar de su salud y bienestar, capacitando e instruyendo al personal sobre buenas prácticas ergonómicas.

\section{Referencias bibliográficas.}

Cuixart, S. N. (2001). NTP 601: Evaluación de las condiciones de trabajo: carga postural. Método REBA (Rapid Entire BodyAssessment),. INSHT, 2. Obtenido de http://www.insht.es/InshtWeb/Contenidos/Documentacion/FichasTecnicas/NTP/Fic heros/601a700/ntp_601.pdf

INSSBT. (s.f.). Check liist para la identificación de las posturas de trabajo forzadas. Obtenido de INSHT:

Sociales, M. d. (2004). Real Decreto 486/1997, de 14 de abril, por el que se establecen las disposiciones mínimas de seguridad y salud en los lugares de trabajo. BOLETÍN OFICIAL DEL ESTADO.

INSSBT. (s.f.). Bursitis prepatelar. Obtenido de Portal de TME : http://www.insht.es/MusculoEsqueleticos/Informacion\%20estructural/TrastornosFr ecuentes/extremidades\%20inferiores\%20Ficheros/Bursitis_Prepatelar.pdf

INSSBT. (s.f.). Epicondilitis. INSSBT, 1.

INSSBT. (s.f.). Epitrocleitis. Obtenido de Portal de Trastornos Musculoesqueléticos: http://www.insht.es/MusculoEsqueleticos/Informacion\%20estructural/TrastornosFr ecuentes/extremidades\%20superiores/ficheros/Epitrocleitis.pdf

INSSBT. (s.f.). Ganglion . Obtenido de Portal de TME: http://www.insht.es/MusculoEsqueleticos/Informacion\%20estructural/TrastornosFr ecuentes/extremidades\%20superiores/ficheros/Ganglion.pdf 
INSSBT. (s.f.). Lumbalgia . Obtenido de Portal TME : http://www.insht.es/MusculoEsqueleticos/Informacion\%20estructural/TrastornosFr ecuentes/espalda/ficheros/Lumbalgia.pdf

INSSBT. (s.f.). Síndrome cervical por tensión. Obtenido de Portal de TME : http://www.insht.es/MusculoEsqueleticos/Informacion\%20estructural/TrastornosFr ecuentes/espalda/ficheros/Sindrome_Tension_Cervical.pdf

INSSBT. (s.f.). Síndrome del Túnel Carpiano. Obtenido de PORTAL DE TME: http://www.insht.es/MusculoEsqueleticos/Informacion\%20estructural/TrastornosFr ecuentes/extremidades\%20superiores/ficheros/Sindrome_Tunel_Carpiano.pdf

INSSBT. (s.f.). Tendinitis del manguito de los rotadores. INSSBT , párr. xx.

Sagrario Cilveti Gubía, Víctor Idoate García. (2000). POSTURAS FORZADAS. COMISIÓN DE SALUD PÚBLICA, 13,14.

Trabajo, I. N. (s.f.). Análisis de posturas forzadas. Recuperado el 18 de 11 de 2017, de http://calculadores.insht.es:86/An\%C3\%A1lisisdeposturasforzadas/Introducci\%C3 $\%$ B3n.aspx

Trabajo, I. N. (s.f.). PORTAL DE TRASTORNOS MUSCULOESQUELETICOS . Recuperado $\begin{array}{lllllll}\text { el } & 18 & \text { de } & 11 & \text { de } & 2017,\end{array}$ http://www.insht.es/MusculoEsqueleticos/Contenidos/Factores\%20de\%20riesgo/Po sturas\%20forzadas/30.Identificacion\%20y\%20ejemplo\%20PF.pdf

OHSAS 18001. (2007). Sistema de Gestión en Seguridad y Salud.pag 16,18

PANEQUE, R. J. (1988). Metologia de la investigacion .

Sagrario Cilveti Gubía, Víctor Idoate García. (2000). POSTURAS FORZADAS. COMISIÓN DE SALUD PÚBLICA, 13,14. 


\section{ANEXO 1.}

\section{CHECK LIST PARA LA IDENTIFICACIÓN DE POSTURAS FORZADAS,}

1. Cabeza en postura forzada:

\section{Girada}

$\square \quad$ Inclinada hacia delante (en flexión acusada)

$\square \quad$ Inclinada hacia atrás

$\square$ Inclinada hacia los lados
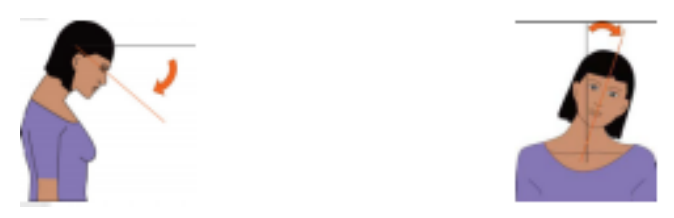

2. Tronco en postura forzada:

Girado

Inclinado hacia delante (en flexión acusada)

Inclinado hacia atrás

Inclinado hacia los lados
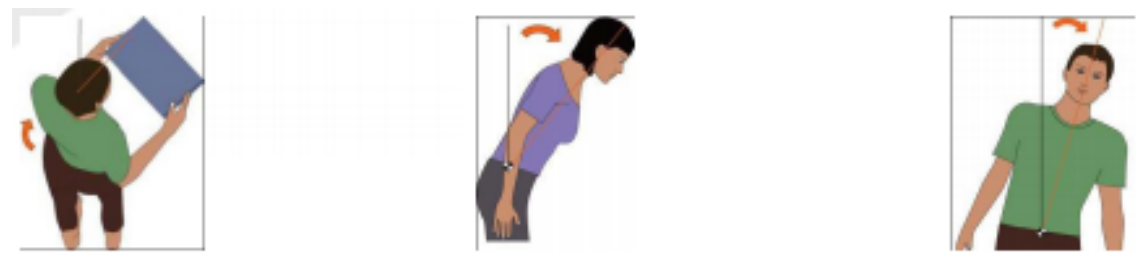

3. Brazo (hombro) en postura forzada:

Brazo levantado hacia delante (flexión acusada)

Brazo hacia atrás del cuerpo (extensión)

Brazo levantado hacia los lados (abducción muy acusada)

Brazo cruzando por delante del cuerpo (aducción)

$\square$ Hombro levantado 

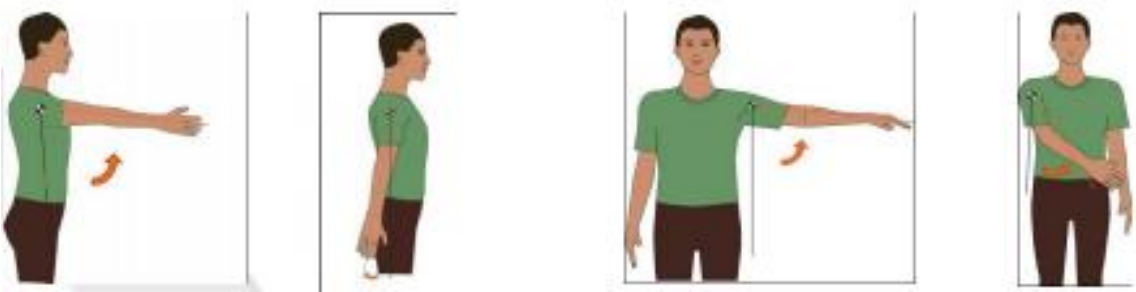

4. Antebrazo (codo) en postura forzada:

Codo muy flexionado

\section{Codo completamente extendido}

Antebrazo en pronación máxima (palma de la mano hacia abajo)

Antebrazo en supinación (palma de la mano hacia arriba)
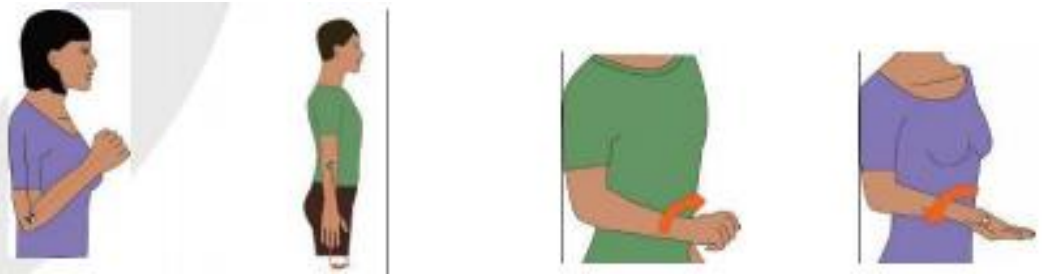

5. Mano (muñeca) en postura forzada:

Muñeca muy flexionada

Muñeca muy extendida

Desviación radial de la mano

Desviación cubital de la mano
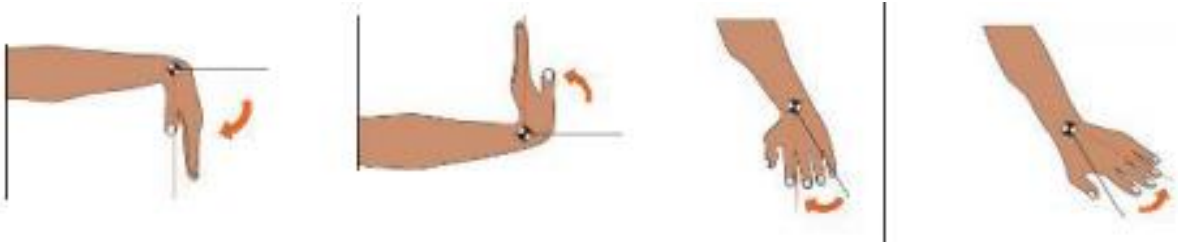

6. Extremidad inferior en postura forzada

Rodillas flexionadas estando de pie

Rodillas muy flexionadas por estar en cuclillas, arrodillado...

Rodillas muy flexionadas estando sentado (pies hacia atrás)

Rodillas muy extendidas estando sentado (sin inclinar el tronco hacia atrás)

Tobillos en flexión (punta del pie hacia abajo) o dorsiflexión (punta del pie hacia arriba) 


\section{ANEXO 2.}

\section{TABLAS DE METODOLOGIA REBA}

Tabla 5. Tabla A carga / fuerza

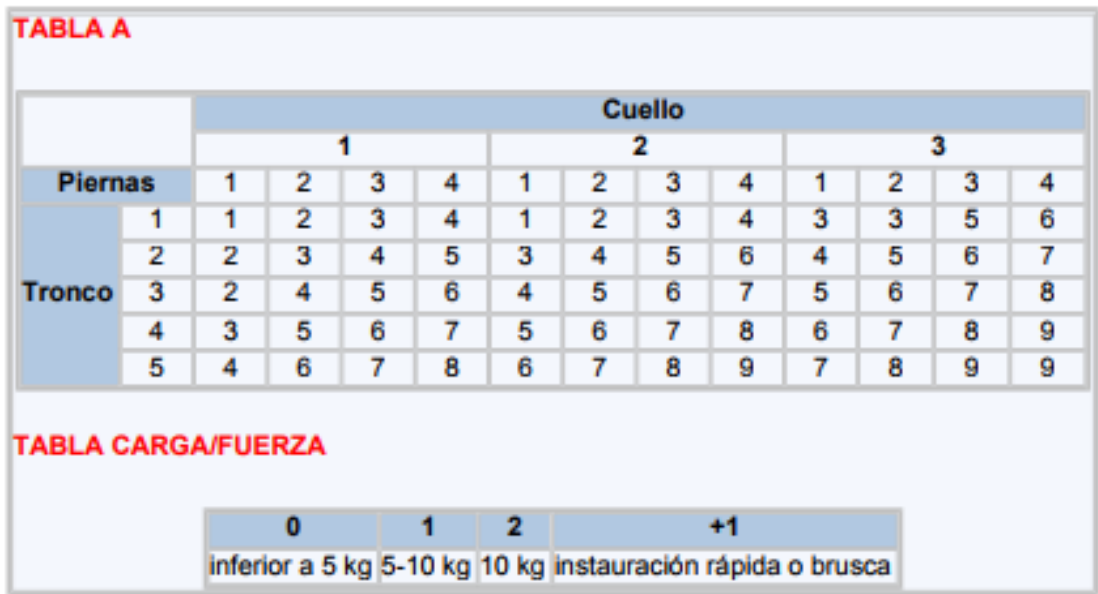

Fuente: NTP 601, 2001

Tabla B / tabla agarre

Tabla 6. Tabla B / tabla agarre

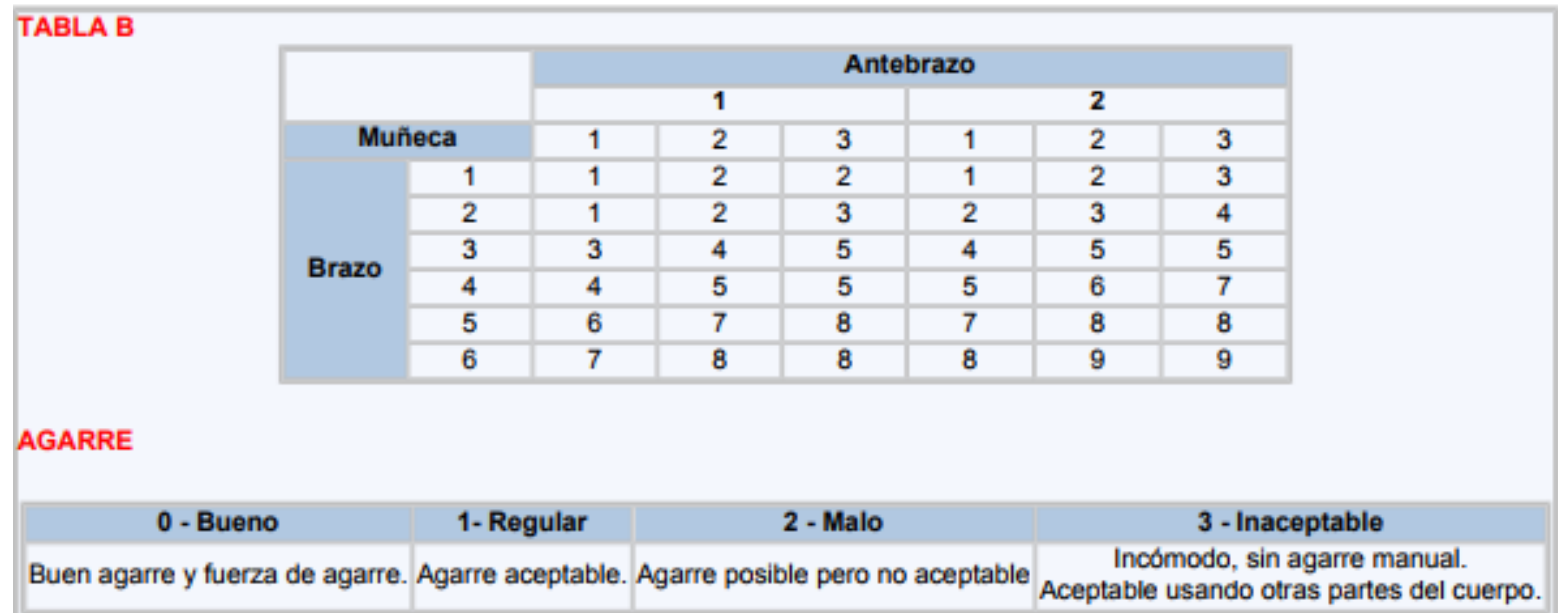

Fuente: NTP 601, 2001 


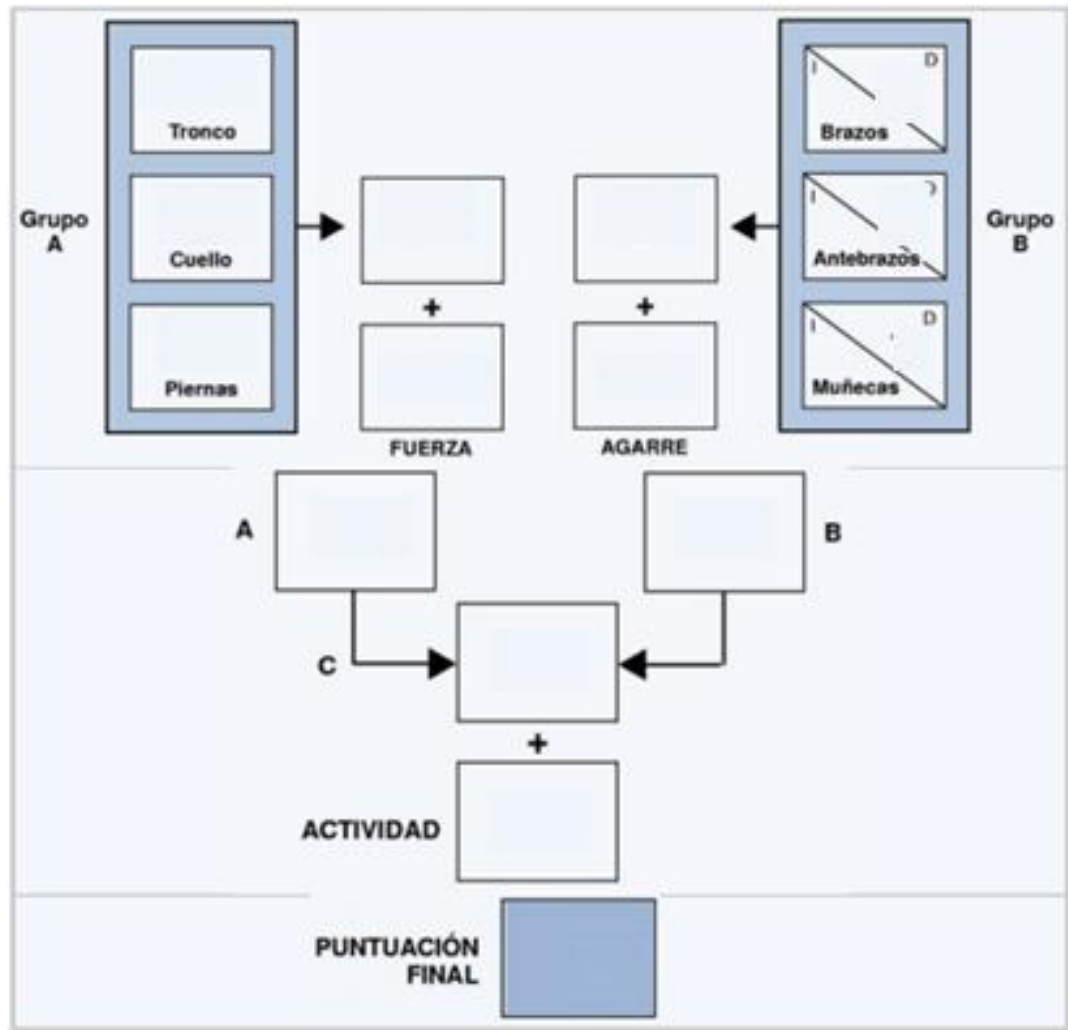

Figura 4. Hoja de puntuación

Hignett, S., McAtamney, L. (2000) 


\section{PARA CITAR EL ARTÍCULO INDEXADO.}

Autor1., Autor2., Autor3., \& Autor4. (2019). Tema del artículo........... Revista electrónica Ciencia Digital 3(2), 78-97. Recuperado desde: http://cienciadigital.org/revistacienciadigital2/index.php/CienciaDigital/article/view/263/56 $\underline{7}$

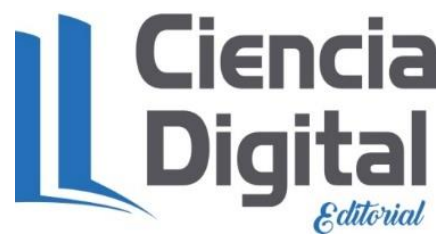

El artículo que se publica es de exclusiva responsabilidad de los autores y no necesariamente reflejan el pensamiento de la Revista Ciencia Digital.

El artículo queda en propiedad de la revista y, por tanto, su publicación parcial y/o total en otro medio tiene que ser autorizado por el director de la Revista Ciencia Digital.
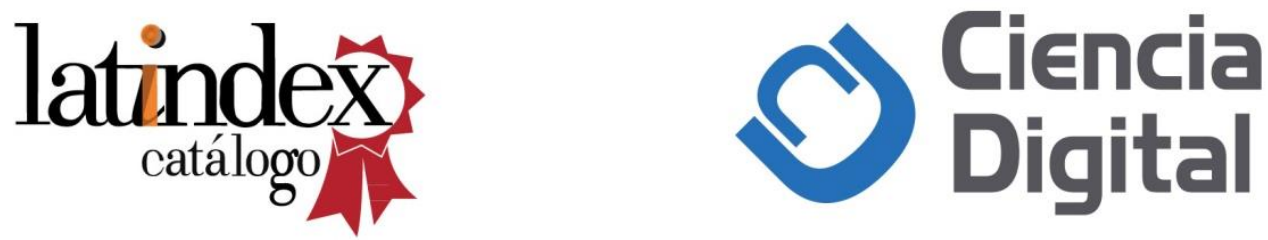